\title{
Article \\ Absence of Host-Specific Genes in Canine and Human Staphylococcus pseudintermedius as Inferred from Comparative Genomics
}

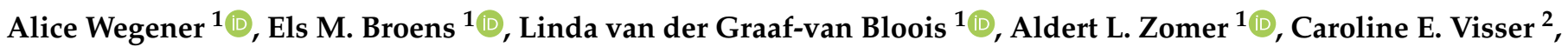 \\ Jan van Zeij1 ${ }^{3}$, Coby van der Meer ${ }^{3}$, Johannes G. Kusters ${ }^{4}$, Alex W. Friedrich ${ }^{5}$, Greetje A. Kampinga ${ }^{5}$, \\ Gregorius J. Sips ${ }^{6,7}$, Leonard Smeets ${ }^{6}$, Manfred E. J. van Kerckhoven ${ }^{8}$, Arjen J. Timmerman ${ }^{1}$, \\ Jaap A. Wagenaar ${ }^{1,9}$ and Birgitta Duim ${ }^{1, *}$
}

check for updates

Citation: Wegener, A.; Broens, E.M.; van der Graaf-van Bloois, L.; Zomer, A.L.; Visser, C.E.; van Zeijl, J.; van der Meer, C.; Kusters, J.G.; Friedrich,

A.W.; Kampinga, G.A.; et al. Absence of Host-Specific Genes in Canine and Human Staphylococcus pseudintermedius as Inferred from Comparative Genomics. Antibiotics 2021, 10, 854. https://doi.org/ 10.3390/antibiotics 10070854

Academic Editors: John Barlow and Laura Selva Martínez

Received: 29 April 2021

Accepted: 30 June 2021

Published: 14 July 2021

Publisher's Note: MDPI stays neutral with regard to jurisdictional claims in published maps and institutional affiliations.

Copyright: (C) 2021 by the authors Licensee MDPI, Basel, Switzerland. This article is an open access article distributed under the terms and conditions of the Creative Commons Attribution (CC BY) license (https:// creativecommons.org/licenses/by/ $4.0 /)$.
1 Department of Infectious Diseases and Immunology, Faculty of Veterinary Medicine, Utrecht University, Yalelaan 1, 3584 CL Utrecht, The Netherlands; a.c.h.wegener@uu.nl (A.W.); E.M.Broens@uu.nl (E.M.B.); l.vandergraaf@uu.nl (L.v.d.G.-v.B.); a.l.zomer@uu.nl (A.L.Z.); a.j.timmerman@uu.nl (A.J.T.); J.Wagenaar@uu.nl (J.A.W.)

2 Amsterdam UMC Location AMC, Meibergdreef 9, 1105 AZ Amsterdam, The Netherlands; c.e.visser@amsterdamumc.nl

3 IZORE Centre for Infectious Diseases Friesland, 8900 JA Leeuwarden, The Netherlands; j.van.zeijl@izore.nl (J.v.Z.); c.vandermeer@izore.nl (C.v.d.M.)

4 Utrecht Medical Center, Heidelberglaan 100, 3584 CX Utrecht, The Netherlands; hans@hanskusters.nl

5 Department of Medical Microbiology, University Medical Center Groningen, University of Groningen, 9700 RB Groningen, The Netherlands; alex.friedrich@umcg.nl (A.W.F.); g.a.kampinga@umcg.nl (G.A.K.)

6 Reiner Haga MDC, Reinier de Graafweg 7, 2625 AD Delft, The Netherlands; g.sips@rivm.nl (G.J.S.); 1.smeets@reiner-mdc.nl (L.S.)

7 Erasmus University Medical Centre (Erasmus MC), Department of Medical Microbiology and Infectious Diseases, 3015 GD Rotterdam, The Netherlands

8 LabMicta, LabMicTA Boerhaavelaan 59, 7555 BB Hengelo, The Netherlands; m.vankerckhoven@labmicta.nl

9 Wageningen Bioveterinary Research, 8200-8249 Houtribweg, 8221 RA Lelystad, The Netherlands

* Correspondence: b.duim@uu.nl

Abstract: Staphylococcus pseudintermedius is an important pathogen in dogs that occasionally causes infections in humans as an opportunistic pathogen of elderly and immunocompromised people. This study compared the genomic relatedness and antimicrobial resistance genes using genome-wide association study (GWAS) to examine host association of canine and human S. pseudintermedius isolates. Canine $(n=25)$ and human $(n=32)$ methicillin-susceptible S. pseudintermedius (MSSP) isolates showed a high level of genetic diversity with an overrepresentation of clonal complex CC241 in human isolates. This clonal complex was associated with carriage of a plasmid containing a bacteriocin with cytotoxic properties, a CRISPR-cas domain and a pRE25-like mobile element containing five antimicrobial resistance genes. Multi-drug resistance (MDR) was predicted in 13 (41\%) of human isolates and 14 (56\%) of canine isolates. CC241 represented 54\% of predicted MDR isolates from humans and $21 \%$ of predicted MDR canine isolates. While it had previously been suggested that certain host-specific genes were present the current GWAS analysis did not identify any genes that were significantly associated with human or canine isolates. In conclusion, this is the first genomic study showing that MSSP is genetically diverse in both hosts and that multidrug resistance is important in dog and human-associated S. pseudintermedius isolates.

Keywords: S. pseudintermedius; comparative genomics; host association; antimicrobial resistance

\section{Introduction}

Staphylococcus pseudintermedius is found both as a commensal bacterium as well as an opportunistic pathogen in dogs. S. pseudintermedius in dogs is associated with skin, soft tissue and systemic infections similar to $S$. aureus infections in humans. Over the last 
decades, S. pseudintermedius is increasingly recognized as a potential zoonotic pathogen of canine origin in elderly and immunocompromised humans [1]. There is an increase in reports of S. pseudintermedius infections in humans, which might be at least partially explained by the implementation of MALDI-TOF MS in routine diagnostics facilitating proper identification of coagulase-positive staphylococci [2-5].

The epidemiology of $S$. pseudintermedius in human infections is poorly studied. Human infections have been reported to be mainly caused by methicillin-susceptible $S$. pseudintermedius (MSSP). This is often thought to result from transmission of MSSP between dogs and humans within the same household [6,7]. Transmission of methicillin-resistant S. pseudintermedius (MRSP) between dogs has been frequently observed, leading to long time carriage with possible re-infections. Dog-to-human transmission was infrequent and no long-term carriage in humans was observed [8]. A single case of human-to-human MRSP-transmission has been described [9].

In contrast with $S$. aureus which has been isolated from multiple host species and shows frequent acquisition, or loss of host-associated genes [10], S. pseudintermedius seems to be more host-restricted. However, information on the genetic variation and the mechanisms that allow adaption of MSSP to humans is scarce. A study on S. pseudintermedius adherence properties to corneocytes revealed a general preference for canine corneocytes compared to human corneocytes [11] and another study identified a cell-wall-associated protein with high binding strength to canine fibrinogen compared to fibrinogen of other host species [12]. In order to identify potential host-specific genes and clones we performed comparative genomics of methicillin-susceptible $S$. pseudintermedius from canine and human origin.

\section{Results}

\subsection{MSSP-Infections Determinants}

Patient information was incomplete for $7 / 32(22 \%)$ of the obtained human isolates. Most human MSSP isolates were from wound infections $(n=18)$. Other infections were ear $(n=2)$, joint $(n=2)$, skin $(n=2)$, systemic $(n=1)$, urinary tract $(n=1)$ and rectum infections $(n=1)$. The age of patients from 24 cases varied between 48 and 86 years; 1 patient was 6 years old (Supplementary Table S1). Patient isolates were obtained from hospitals located in six different provinces. The canine MSSP isolates were isolated from different body sites. The majority of canine isolates were from ear infections $(n=9)$, followed by skin infections $(n=8)$, wound infections $(n=5)$, urinary tract infections $(n=2)$ and a joint infection $(n=1)$ (Supplementary Table S1). All isolates were obtained between 2014 and January 2019. Isolates were isolated from different patients at different time points and are to the best of our knowledge unrelated epidemiologically.

\subsection{S. pseudintermedius Phylogeny}

The genetic relatedness of canine and human MSSP isolates is visualized in a phylogenetic SNP tree of the core genome (size of 2,170,170 bp) in comparison with included publicly available genomes [12-14] (Figure 1). This placed our results in a wide epidemiological context and shows that the isolates of this study are dispersed among the high genomic diversity of MSSP isolates.

To zoom in on the genome comparison of the dog and human isolates from this study the genotype details are shown in a phylogenetic tree in Figure 2. The extracted MLST types were superimposed, and the studied isolates belonged to 50 different sequence types (ST). The observed phylogenetic diversity between MSSP genomes was high overall and showed 1 cluster containing 10 genomes. This cluster, corresponding to clonal complex CC241 (comprising of ST241, ST941, ST1379, ST1350, ST1360), dominated, and consisted of seven human isolates (comprising of ST241, ST941, ST1379) and three canine isolates (comprising of ST241, ST1350, ST1360). While CC241 was overrepresented in humans, the difference in proportion of isolates from both hosts present in the CC241 cluster was not statistically significant ( $p=0.487$ ). Besides the genome cluster belonging to CC241, only two other genetically related pairs were identified, with 1 pair consisting of a canine and a 
human isolate of ST989 with 55 SNP differences and 1 pair with 2 human isolates of ST985 from the same hospital with 8 SNP differences in their core genome. All other STs were represented by a single isolate (Figure 2).

Tree scale: 1000

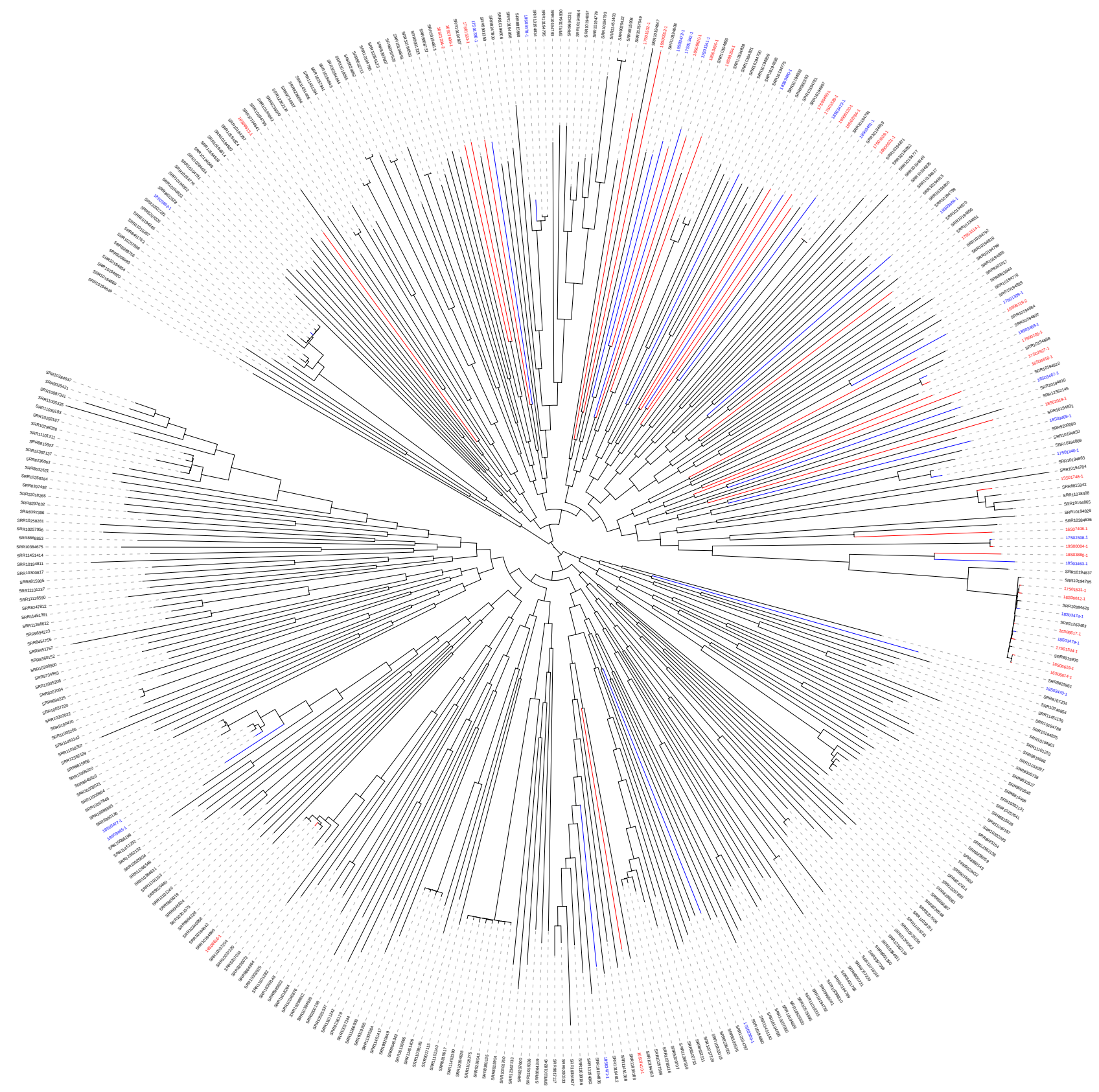

Figure 1. Phylogenetic tree based on the core genome SNPs of MSSP isolates. Publicly available MSSP genomes were compared with the MSSP isolates from this study, that have been marked red for human isolates and blue for dog isolates [12-14]. 


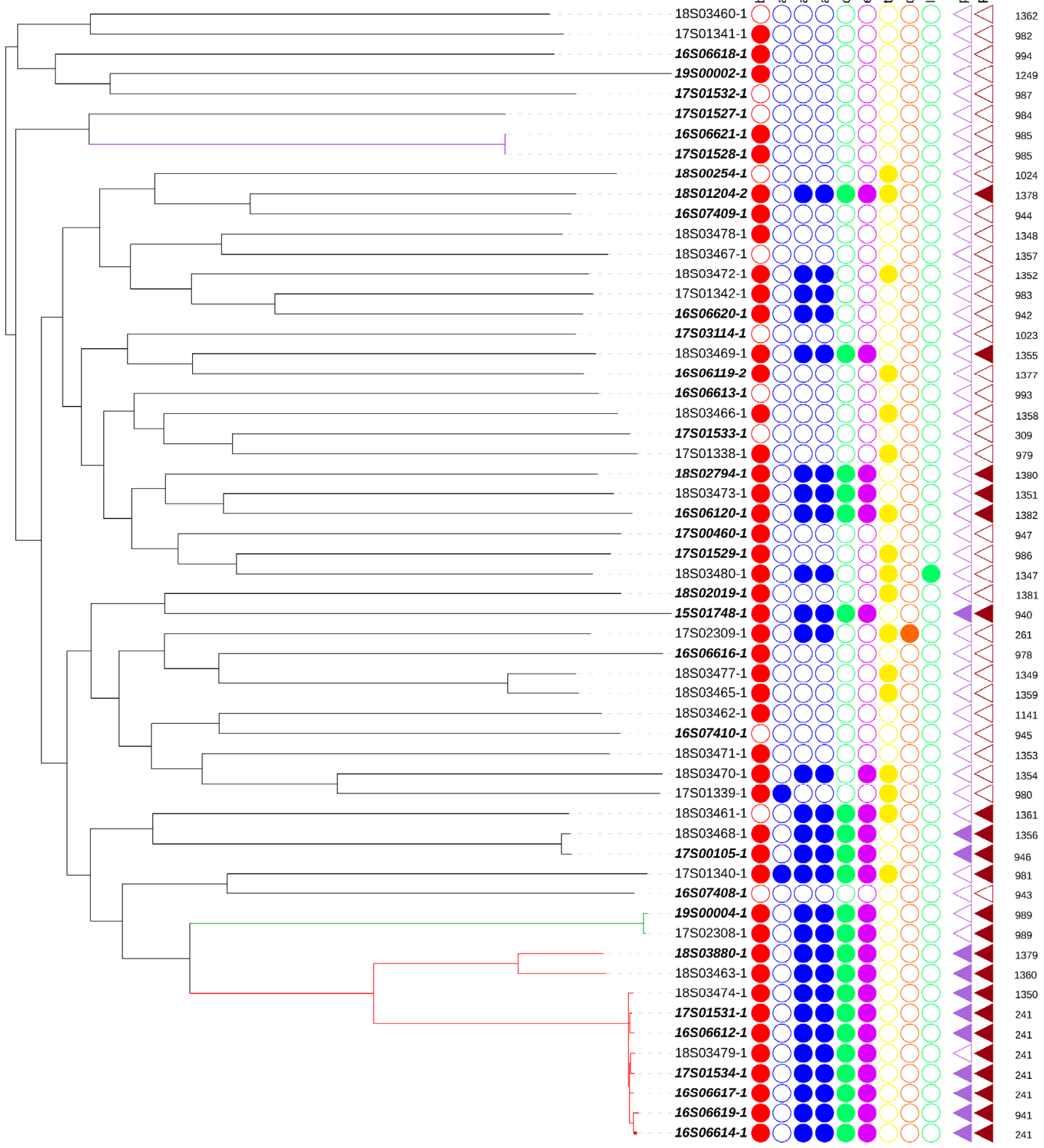

Figure 2. Isolate phylogeny and antimicrobial resistance genes.

Figure 2 shows a core-genome SNP tree of canine and humans (bold italic) isolates, presence (filled circles) or absence (empty circles) of antimicrobial resistance genes, presence of mobile genetic element PRE25-like [15] and plasmid P222 [16] (filled triangles), and sequence type (ST). Clones are shown by coloured branches (CC241 in red, ST989 in green, ST985 in purple). The length of the branch represents the number of SNPs.

\subsection{Antimicrobial Resistance Genes}

In S. pseudintermedius it has been shown that antimicrobial resistance genotypes can accurately predict phenotypical antimicrobial resistances $[14,17,18]$. Multidrug resistance 
(MDR) (i.e., carriage of resistance genes to 3 or more classes of antimicrobials) was identified in 13/32 (41\%) human isolates and 14/25 (56\%) canine isolates (Supplementary Table S1, Figure 1 and Table 1). No resistance genes were identified using Resfinder in 7/32 (22\%) human isolates and in 2/25 (8\%) canine isolates. Following the statistical analysis for host association, no statistically significant difference was found neither for the prevalence of predicted MDR in human versus canine isolates $(p=0.249)$, nor for isolates without resistance genes $(p=0.273)$.

Table 1. Antimicrobial resistance genes in human and canine S. pseudintermedius isolates.

\begin{tabular}{|c|c|c|c|}
\hline Resistance & Gene & $\begin{array}{l}\text { Human Isolates } \\
\quad(n=32)\end{array}$ & $\begin{array}{c}\text { Canine Isolates } \\
\quad(n=25)\end{array}$ \\
\hline$\beta$-lactam & blaZ & $25(78 \%)$ & $23(92 \%)$ \\
\hline \multirow{2}{*}{ aminoglycoside } & ant6-Ia, aph $\left(3^{\prime}\right)-I I I$ & $14(44 \%)$ & $14(56 \%)$ \\
\hline & $a a c\left(6^{\prime}\right)-I e-a p h\left(2^{\prime \prime}\right)-I a$ & 0 & $2(8 \%)$ \\
\hline chloramphenicol & $\operatorname{cat}_{(\mathrm{pC} 221)}$ & $13(41 \%)$ & $9(36 \%)$ \\
\hline macrolide & $\operatorname{erm}(B)$ & $13(41 \%)$ & $10(40 \%)$ \\
\hline tetracycline & $\operatorname{tet}(M)$ & $6(19 \%)$ & $11(44 \%)$ \\
\hline lincosamide & $\operatorname{Inu}(A)$ & 0 & $1(4 \%)$ \\
\hline folate inhibitor & $d f r G$ & 0 & $1(4 \%)$ \\
\hline
\end{tabular}

The distribution of the identified antimicrobial resistance genes in MSSP from both hosts is presented in Table 1 . The penicillin resistance gene blaZ was found in the majority of isolates from both hosts (48/57) (84\%). Aminoglycoside resistance genes ant6-Ia and aph $\left(3^{\prime}\right)$-III were found in 14/25 (56\%) of the canine isolates compared to 14/32 (44\%) in human isolates. The gene $a a c\left(6^{\prime}\right)-I e-a p h\left(2^{\prime \prime}\right)-I a$ was detected in two canine isolates. The chloramphenicol resistance gene cat $_{(\mathrm{pC} 221)}$ was present in 13/32 (41\%) of human isolates and $9 / 25(36 \%)$ of canine isolates. The macrolide resistance gene $\mathrm{erm}(B)$ was present in 13/32 (41\%) of human isolates and 10/25 (40\%) of canine isolates. The tetracycline resistance gene tet $(M)$ was present in $11 / 25(44 \%)$ of the canine isolates and 6/32 (19\%) of the human isolates. The folate pathway inhibitor resistance gene $d f r G$ and lincosamide resistance gene $\operatorname{Inu}(A)$ were not present in human isolates and only in $1 / 25(4 \%)$ of the canine isolates. No statistically significant host association was found for the differences in resistance gene presence in canine and human isolates.

\subsection{Host-Associated Genes}

We did not identify gene presence or absence significantly associated with either canine or human S. pseudintermedius isolates using Roary (Table S2).

The genes spsL and spsD were studied specifically using alignments, as they were previously linked with host specificity $[11,19]$. The complete gene encoding the fibrinogen binding protein spsD was detected in 25 human isolates and 21 canine isolates. The fibronectin and fibrinogen binding protein spsL was present in 30 human isolates and 24 canine isolates. Alignment to reference genes spsD and spsL from ED99 showed a high level of sequence diversity across the gene with no clear clustering of variants of either dogs or human isolates (Figures S1 and S2).

As CC241 was overrepresented in human isolates, a GWAS study on orthologs associated with this clonal complex was performed that identified several genes shown in Table S3. In summary, 9 out of 10 CC241 isolates carried the plasmid p222 (99\% identity and $97 \%$ coverage with the reference plasmid) encoding a bacteriocin with cytotoxic effect that was previously identified in a canine $S$. pseudintermedius isolate [16]. This p222 plasmid was also present in three (6\%) non-CC241 isolates (two human (ST940 and ST946) and one canine (ST1356) isolate). The pRE25-like element that has been described as a chromosomal element carrying five resistance genes; $\operatorname{erm}(B), \operatorname{cat}(p C 221)$, aph (3')-III, ant6-Ia, sat4 and a toxin antitoxin system [15] was also associated with CC241 isolates. Sequence homology $(>90 \%)$ with this element was detected in all CC241 isolates $(n=10)$ as well as in seven 
non-CC241 human isolates belonging to other sequence types belonging to ST940, ST946, ST989, ST1378, ST1380, ST1382, and in six non-CC241 canine isolates belonging to ST981, ST989, ST1351, ST1355, ST1356, and ST1361. Despite the sequence homology, all isolates missed one or more transposase genes (Table S3). Manual investigation of the sequence assembly graphs using Bandage [20] revealed that collapsed repeats were the reason for these missing genes, a common issue with assembly from short-read sequences.

Next to the p222 plasmid and the pRE25-like element, a CRISPR-cas-type-III region was associated with CC241 and present in nine out of ten CC241 isolates. This CRISPR type was present as well in three other human isolates belonging to ST309, ST943 and ST946. All genes associated with either presence or absence in CC241 in the GWAS analysis are presented in Table S3.

\section{Discussion}

Until a decade ago, human infections with $S$. pseudintermedius were only seldom reported, but the number of reports has markedly increased over the last decade, which could partially be linked to the use of MALDI-TOF [2-5]. Although zoonotic transmission from dogs to humans is generally suspected very few studies have compared the genetic characteristics of $S$. pseudintermedius isolates originating from human infections versus canine sources.

This study revealed that CC241 was overrepresented in the studied MSSP isolates and was composed mainly of human isolates. It is of interest to monitor the MDR CC241 clone as it carries genetic elements which can confer a number of selective advantages. First, the identification of a CRISPR-cas system associated with CC241 might increase genetic stability of CC241 which was shown for MRSP earlier [18], but not previously observed in MSSP. A second potential selective advantage could be the association of CC241 with plasmid p222, which contains BacSp222, a multifunctional peptide that functions as a bacteriocin against Gram-positive bacteria and is a virulence factor affecting eukaryotic cells [16]. A third potential selective advantage could be the multidrug resistance provided by the pRE25-like mobile element carrying five resistance genes coding for resistance to four antimicrobial classes [15]. This element with the IS1216 transposase on both ends is highly related to the pRE25 plasmid of Enterococcus faecalis and has recently been detected in canine isolates of S. pseudintermedius in Korea [15]. In the present study this pRE25-like element was present in all CC241 isolates, but was not limited to this clone, as it was present in 12 non-CC241 isolates as well. In the Korean study the STs of pRE25-like element containing isolates were diverse, but a selective advantage leading to dominance of clones containing this element was suggested [15]. To study in more detail the dissemination of MDR and the pRE25-like element we compared our isolate with two large studies with published canine MSSP genomes. The first one was in Europe where $8 \%$ of MSSP isolates were MDR and none had genes associated with pRE25-like $\left(\operatorname{erm}(B), \operatorname{cat}_{(p C 221)}\right.$, aph $\left(3^{\prime}\right)-I I I$, ant6-Ia, sat4) [13]. The second in the USA showed 22.1\% MDR among isolates containing $\operatorname{erm}(B), c_{(p C 221)}$, aph $\left(3^{\prime}\right)$-III, ant6-Ia, sat4, and 11 isolates carried the pRE25-like element [14]. Alignment with this element showed high homology with the pRE25-like elements identified in CC241 isolates in this study, and 4/11 isolates belonged to CC241 and the others belonged to various sequence types. The multiple findings of the pRE25-like element indicates that it is present in distinct MSSP lineages and most likely contributes to dissemination of resistance genes in MSSP, in the same way as has been observed for the resistance element carrying Tn5405 in MRSP [21]. The carriage of each of these elements will result in multidrug resistance and could limit treatment options for MSSP infections. The spread of this element among other human isolates could not be studied as none of the four publicly available MSSP genomes were multidrug resistant [12]. A similar finding of low phenotypic multidrug resistance ( 2 of the 24 tested isolates) has been reported for other human MSSP isolates [6].

Furthermore, we observed a high diversity in MSSP amongst both canine and human isolates, which confirms the diversity reported for canine MSSP isolates in a core genome 
MLST analysis [13]. To exclude that geographical differences are responsible for the observed diversity, a SNP phylogeny with publicly available MSSP genomes and our isolates was constructed. This confirmed the high diversity with small lineages, indicating that there is no geographical cluster of Dutch isolates, as they were dispersed over the tree. It also indicated that the lineage associated with CC241 was still overrepresented in human isolates $[13,14]$ (Figure S3). This high diversity in MSSP is in contrast with the observed clonal dissemination of MRSP [13]. The phylogeny based on SNPs in the MSSP core genomes identified a deep-branched structure corresponding with a distinct MLST type for almost all isolates, whereas MRSP isolates mainly belong to a limited number of clonal complexes [13]. The genetic stability within certain MRSP clones might be explained by the carriage of lineage-specific prophages, restriction-modification or CRISPR/Cas systems hindering DNA uptake [18]. Furthermore, MRSP isolates often harbour resistance genes to multiple antimicrobial classes and use of any antimicrobial might co-select for the spread of these MDR isolates [21,22]. These elements might also explain the dominance of CC241 in our selection of MSSP isolates as all CC241 isolates were predicted MDR and contained lineage-specific elements, but were remarkably mainly found in human isolates (7/10). CC241 has also been described in a human infection in Spain [7], indicating a wider presence which is noticeable given the genetic diversity of MSSP.

Currently the number of $S$. pseudintermedius genomes from human infections is very low. This study provides the first genome comparison of human MSSP isolates, and analysis of more human isolates will be needed to unravel the importance of CC241 or other lineages in human MSSP infections.

GWAS analysis based on gene presence/absence showed no host association of specific genes which could be explained by the limited sample size but could also be due to the absence of host adaptation. The high sequence diversity of MSSP found in both sources might also play a role in the difficulty of finding host association. As an example, the genes encoding fibrinogen binding proteins (spsD and spsL) that were previously shown to be host associated $[11,19]$ showed a high level of diversity, in both canine and human isolates (Figures S1 and S2). While these genes have been reported to contain repeat regions which might account for some diversity [23], in our isolates genetic variation was not limited to those regions. This variability in fibronectin binding protein encoding genes has not been previously reported. Studying allele variants rather than analysing gene presence/absence could be necessary to identify host-associated genes using, e.g., k-mer approaches. Several hundred isolates would probably be required to detect statistically significant associations because of the more severe multiple testing correction, as was shown when evaluating a k-mer-based approach in Streptococcus pneumoniae [24]. Analysis of protein variants or differences in protein domains, such as has been observed for leucocidins in S. aureus and S. pseudintermedius $[25,26]$, was beyond the scope of this study.

No statistically significant difference was found between dog and human isolates in the carriage of resistance genes. Multidrug resistance was present in $41 \%$ of human isolates and $56 \%$ of canine isolates. The tet $(M)$ gene was found slightly more often in dogs than in humans even though this was not statistically significant. Resistance genes, not located on pRE25-like elements, encoding for resistance against aminoglycosides (aac $\left(6^{\prime}\right)$-Ie$\left.\operatorname{aph}\left(2^{\prime \prime}\right)-I a\right)$, lincosamides $(\operatorname{Inu}(A))$ and folate pathway inhibitors $(\operatorname{dfr} G)$, were only found in non-CC241 canine isolates. However, the sample size of human isolates was rather small. The prevalence of these genes was low in canine isolates which might explain why these genes were not identified in human isolates in this study.

\section{Materials and Methods}

\subsection{Bacterial Isolates}

In total, 57 MSSP isolates were included, 32 S. pseudintermedius isolates from human infections obtained from 6 Dutch hospitals between 2014 and January 2019, and 25 S. pseudintermedius isolates from canine infections isolated at the Veterinary Microbiological Diagnostic Centre were selected to match the years of isolation of human isolates. All isolates 
were selected based on convenience sampling and epidemiologically unrelated. Species identification was confirmed by matrix-assisted laser desorption ionization time-of-flight (MALDI-TOF MS) (Bruker MALDI Biotyper, Bruker Daltonics, Billerica, MA, USA).

\subsection{Genome Analysis}

DNA was isolated using the Qiagen UltraClean Microbial DNA isolation kit (Qiagen, venlo, the Netherlands. DNA libraries were prepared with the Illumina Nextera kit according to manufacturer's instructions and sequenced using NextSeq sequencing with 150 base pairs reads (Illumina, San Diego, CA, USA). Reads-quality-check and adaptertrimming was performed with Trim Galore v0.4.4 (https: / / www.bioinformatics.babraham. ac.uk/projects /trim_galore/) Last update: 24 March 2017. The genomes were assembled with SPAdes v3.10.1 [27], and contigs smaller than 200 base pairs and with a coverage lower than 10 were removed. Genome quality was assessed with CheckM v1.1.2 [28] for completeness $(>95 \%)$ and contamination $(<5 \%)$. The genomes were annotated using Prokka v1.13 [29]. The batch upload function, including Resfinder and MLSTFinder, from the Center for Genomic Epidemiology (CGE) (Copenhagen, Denmark) [30] was used to analyse the resistance gene content and sequence types of all isolates (last accessed on 12 March 2019). In case of new sequence types, the alleles for Multi Locus Sequence Type (MLST) were assigned an ST number by the curator of the PubMLST database (https:/ / pubmlst.org/spseudintermedius/) (accessed on 5 March 2019).

The whole genome sequences were aligned, and the core genome size determined, using Parsnp v1.2 [31] for phylogenetic single-nucleotide polymorphism (SNP) analysis of the core genome that was visualised using ITOL v4 [32] and a minimum spanning tree was made with Phyloviz 2.0 [33] using the goeBURST algorithm to assess the number of single-nucleotide polymorphisms between isolates. Orthology predictions of the annotated genomes were made using Roary 3.12.0 [34] and host- and clonal complex associated genes were determined using Scoary 1.6.16 [35] applying a threshold of $p<0.05$ for statistical significance using Bonferroni correction. Identification of regions with significant genes was performed with a BLAST search against the NCBI database, resulting in the identification of two mobile elements (identity $>90 \%$ coverage $>80 \%$ ). Mobile elements with Genbank accessions CP011490 and MK775653 were used as reference sequences for alignment to the MSSP sequence contigs using Geneious version 2020.1.1 (Biomatters, Auckland, New Zealand). Annotation of the reference element was used to predict the annotation of orthologous genes; if annotation in the reference was absent the annotation of Prokka was used. Statistically significant genes annotated as coding for hypothetical proteins by Prokka were further analysed using NCBI BLASTn 2.10.1. Geneious was also used for the alignment of spsL and spsD genes to their references in ED99 strain CP002478 and for construction of the spsL and spsD trees.

Pearson's chi-square or Fisher's exact test (when sample size $<5$ ) were used for statistical analysis for host association of resistance genes. A $p$-value of $<0.05$ was considered statistically significant with Bonferroni correction.

\subsection{Data Availability}

Whole genome sequence reads and assembled contigs have been deposited in the NCBI Sequence Read Archive under project number PRJEB39511, accession numbers are available in Supplementary Table S1.

\section{Conclusions}

In conclusion, canine and human S. pseudintermedius from the Netherlands were genetically highly diverse, and no host-specific genes could be detected. CC241 was overrepresented in human isolates. The CC241 isolates carried BacSp222, a bacteriocin with cytotoxic activity and the mobile genetic pRE25-like element carrying five resistance genes, which was more widely present in the MSSP population. The dissemination of the 
pRE25-like element could pose a threat for treatment of human and canine S. pseudintermedius infections.

Supplementary Materials: The following are available online at https://www.mdpi.com/article/ 10.3390/antibiotics10070854/s1, Table S1: Isolate information, resistances and mobile elements in human and canine MSSP isolates, Table S2: Roary-Scoary analysis of dog and human isolates. Table S3: Genome wide association study of CC241 defined with Roary-Scoary analysis. Figure S1: Tree of spsD genes of dog and humans. Figure S2: Tree of spsL genes of dog and humans. Figure S3: Phylogenetic tree of MSSP isolates.

Author Contributions: Conceptualization, B.D., E.M.B., J.A.W. and A.W.; methodology, A.J.T.; software, L.v.d.G.-v.B. and A.L.Z.; validation, B.D., E.M.B. and J.A.W.; formal analysis, A.W. and L.v.d.G.v.B.; investigation, A.W.; resources, C.E.V., J.v.Z., C.v.d.M., J.G.K., A.W.F., G.A.K., G.J.S., L.S. and M.E.J.v.K.; data curation, L.v.d.G.-v.B., A.L.Z., A.W. and B.D.; writing—original draft preparation, A.W.; writing—review and editing B.D., E.M.B., J.A.W., J.G.K., A.W. and G.J.S.; visualization, A.W.; supervision, E.M.B., J.A.W. and B.D.; project administration, B.D., E.M.B. and J.A.W. All authors have read and agreed to the published version of the manuscript.

Funding: This research received no external funding.

Institutional Review Board Statement: Not applicable.

Informed Consent Statement: Not applicable.

Data Availability Statement: Sequence reads are available under number PRJEB39511.

Acknowledgments: We thank everyone who participated in the collection of isolates across all participating institutions.

Conflicts of Interest: The authors declare no conflict of interest.

\section{References}

1. Somayaji, R.; Rubin, J.E.; Priyantha, M.A.; Church, D. Exploring Staphylococcus pseudintermedius: An emerging zoonotic pathogen? Future Microbiol. 2016, 11, 1371-1374. [CrossRef]

2. Börjesson, S.; Gómez-Sanz, E.; Ekström, K.; Torres, C.; Grönlund, U. Staphylococcus pseudintermedius can be misdiagnosed as staphylococcus aureus in humans with dog bite wounds. Eur. J. Clin. Microbiol. Infect. Dis. 2014. [CrossRef]

3. Viau, R.; Hujer, A.M.; Hujer, K.M.; Bonomo, R.A.; Jump, R.L.P. Are Staphylococcus intermedius infections in humans cases of mistaken identity? A case series and literature review: Table 1. Open Forum Infect. Dis. 2015, 2, ofv110. [CrossRef]

4. Lee, J.; Murray, A.; Bendall, R.; Gaze, W.; Zhang, L.; Vos, M. Improved detection of Staphylococcus intermedius group in a routine diagnostic laboratory. J. Clin. Microbiol. 2015, 53, 961-963. [CrossRef]

5. Nisa, S.; Bercker, C.; Midwinter, A.C.; Bruce, I.; Graham, C.F.; Venter, P.; Bell, A.; French, N.P.; Benschop, J.; Bailey, K.M.; et al. Combining MALDI-TOF and genomics in the study of methicillin resistant and multidrug resistant Staphylococcus pseudintermedius in New Zealand. Sci. Rep. 2019, 9, 1271. [CrossRef]

6. Somayaji, R.; Priyantha, M.A.R.; Rubin, J.E.; Church, D. Human infections due to Staphylococcus pseudintermedius, an emerging zoonosis of canine origin: Report of 24 cases. Diagn. Microbiol. Infect. Dis. 2016, 85, 471-476. [CrossRef] [PubMed]

7. Lozano, C.; Rezusta, A.; Ferrer, I.; Pérez-Laguna, V.; Zarazaga, M.; Ruiz-Ripa, L.; Revillo, M.J.; Torres, C. Staphylococcus pseudintermedius human infection cases in Spain: Dog-to-human transmission. Vector Borne Zoonotic Dis. 2017, 17, 268-270. [CrossRef]

8. Laarhoven, L.M.; de Heus, P.; van Luijn, J.; Duim, B.; Wagenaar, J.A.; van Duijkeren, E. Longitudinal study on methicillin-resistant Staphylococcus pseudintermedius in households. PLoS ONE 2011, 6, e27788. [CrossRef] [PubMed]

9. Starlander, G.; Börjesson, S.; Grönlund-Andersson, U.; Tellgren-Roth, C.; Melhus, Å. Cluster of infections caused by methicillinresistant Staphylococcus pseudintermedius in humans in a tertiary hospital. J. Clin. Microbiol. 2014, 52, 3118-3120. [CrossRef] [PubMed]

10. Matuszewska, M.; Murray, G.G.R.; Harrison, E.M.; Holmes, M.A.; Weinert, L.A. The evolutionary genomics of host specificity in staphylococcus aureus. Trends Microbiol. 2020, 28, 465-477. [CrossRef]

11. Latronico, F.; Moodley, A.; Nielsen, S.S.; Guardabassi, L. Enhanced adherence of methicillin-resistant Staphylococcus pseudintermedius sequence type 71 to canine and human corneocytes. Vet. Res. 2014, 45, 70. [CrossRef]

12. Little, S.V.; Bryan, L.K.; Hillhouse, A.E.; Konganti, K.; Lawhon, S.D. Whole-genome sequences of Staphylococcus pseudintermedius isolates from canine and human bacteremia infections. Microbiol. Resour. Announc. 2019, 8. [CrossRef]

13. Haenni, M.; el Garch, F.; Miossec, C.; Madec, J.-Y.Y.; Hocquet, D.; Valot, B. High Genetic Diversity among Methicillin-Susceptible Staphylococcus Pseudintermedius in Dogs in Europe. J. Glob. Antimicrob. Resist. 2020, 21, 57-59. [CrossRef] [PubMed] 
14. Tyson, G.H.; Ceric, O.; Guag, J.; Nemser, S.; Borenstein, S.; Slavic, D.; Lippert, S.; McDowell, R.; Krishnamurthy, A.; Korosec, S.; et al. Genomics accurately predicts antimicrobial resistance in Staphylococcus pseudintermedius collected as part of Vet-LIRN resistance monitoring. Vet. Microbiol. 2021, 254, 109006. [CrossRef]

15. Kang, J.H.; Hwang, C.Y. First detection of multiresistance PRE25-like elements from enterococcus spp. in Staphylococcus pseudintermedius isolated from canine pyoderma. J. Glob. Antimicrob. Resist. 2020, 20, 304-308. [CrossRef]

16. Wladyka, B.; Piejko, M.; Bzowska, M.; Pieta, P.; Krzysik, M.; Mazurek, Ł.; Guevara-Lora, I.; Bukowski, M.; Sabat, A.J.; Friedrich, A.W.; et al. A peptide factor secreted by Staphylococcus pseudintermedius exhibits properties of both bacteriocins and virulence factors. Sci. Rep. 2015, 5, 14569. [CrossRef]

17. Wegener, A.; Broens, E.M.; Zomer, A.; Spaninks, M.; Wagenaar, J.A.; Duim, B. Comparative genomics of phenotypic antimicrobial resistances in methicillin-resistant Staphylococcus pseudintermedius of canine origin. Vet. Microbiol. 2018, 225, 125-131. [CrossRef] [PubMed]

18. Brooks, M.R.; Padilla-Vélez, L.; Khan, T.A.; Qureshi, A.A.; Pieper, J.B.; Maddox, C.W.; Alam, M.T. Prophage-mediated disruption of genetic competence in Staphylococcus Pseudintermedius. mSystems 2020, 5. [CrossRef]

19. Pickering, A.C.; Vitry, P.; Prystopiuk, V.; Garcia, B.; Höök, M.; Schoenebeck, J.; Geoghegan, J.A.; Dufrêne, Y.F.; Ross Fitzgerald, J. Host-specialized fibrinogen-binding by a bacterial surface protein promotes biofilm formation and innate immune evasion. PLoS Pathog. 2019, 15, e1007816. [CrossRef] [PubMed]

20. Wick, R.R.; Schultz, M.B.; Zobel, J.; Holt, K.E. Bandage: Interactive visualization of de novo genome assemblies. Bioinformatics 2015, 31, 3350-3352. [CrossRef] [PubMed]

21. McCarthy, A.J.; Harrison, E.M.; Stanczak-Mrozek, K.; Leggett, B.; Waller, A.; Holmes, M.A.; Lloyd, D.H.; Lindsay, J.A.; Loeffler, A. Genomic insights into the rapid emergence and evolution of MDR in Staphylococcus pseudintermedius. J. Antimicrob. Chemother. 2014, 70, 997-1007. [CrossRef] [PubMed]

22. Bannoehr, J.; ben Zakour, N.L.; Waller, A.S.; Guardabassi, L.; Thoday, K.L.; van den Broek, A.H.M.; Fitzgerald, J.R. Population genetic structure of the Staphylococcus intermedius group: Insights into Agr diversification and the emergence of methicillinresistant strains. J. Bacteriol. 2007, 189, 8685-8692. [CrossRef]

23. Bannoehr, J.; Zakour, N.L.B.; Reglinski, M.; Inglis, N.F.; Prabhakaran, S.; Fossum, E.; Smith, D.G.; Wilson, G.J.; Cartwright, R.A.; Haas, J.; et al. Genomic and surface proteomic analysis of the canine pathogen Staphylococcus pseudintermedius reveals proteins that mediate adherence to the extracellular matrix. Infect. Immun. 2011, 79, 3074-3086. [CrossRef] [PubMed]

24. Lees, J.A.; Vehkala, M.; Välimäki, N.; Harris, S.R.; Chewapreecha, C.; Croucher, N.J.; Marttinen, P.; Davies, M.R.; Steer, A.C.; Tong, S.Y.C.; et al. Sequence element enrichment analysis to determine the genetic basis of bacterial phenotypes. Nat. Commun. 2016, 7, 12797. [CrossRef]

25. Koop, G.; Vrieling, M.; Storisteanu, D.M.L.; Lok, L.S.C.; Monie, T.; van Wigcheren, G.; Raisen, C.; Ba, X.; Gleadall, N.; Hadjirin, N.; et al. Identification of LukPQ, a novel, equid-adapted leukocidin of Staphylococcus aureus. Sci. Rep. 2017, 7, 40660. [CrossRef]

26. Abouelkhair, M.A.; Bemis, D.A.; Kania, S.A. Characterization of Recombinant Wild-Type and Nontoxigenic Protein A from Staphylococcus pseudintermedius. Virulence 2018. [CrossRef] [PubMed]

27. Bankevich, A.; Nurk, S.; Antipov, D.; Gurevich, A.A.; Dvorkin, M.; Kulikov, A.S.; Lesin, V.M.; Nikolenko, S.I.; Pham, S.; Prjibelski, A.D.; et al. SPAdes: A new genome assembly algorithm and its applications to single-cell sequencing. J. Comput. Biol. 2012, 19, 455-477. [CrossRef]

28. Parks, D.H.; Imelfort, M.; Skennerton, C.T.; Hugenholtz, P.; Tyson, G.W. CheckM: Assessing the quality of microbial genomes recovered from isolates, single cells, and metagenomes. Genome Res. 2015, 25, 1043-1055. [CrossRef]

29. Seemann, T. Prokka: Rapid prokaryotic genome annotation. Bioinformatics 2014, 30, 2068-2069. [CrossRef] [PubMed]

30. Thomsen, M.C.F.; Ahrenfeldt, J.; Cisneros, J.L.B.; Jurtz, V.; Larsen, M.V.; Hasman, H.; Aarestrup, F.M.; Lund, O. A bacterial analysis platform: An integrated system for analysing bacterial whole genome sequencing data for clinical diagnostics and surveillance. PLoS ONE 2016, 11, e0157718. [CrossRef] [PubMed]

31. Treangen, T.J.; Ondov, B.D.; Koren, S.; Phillippy, A.M. The harvest suite for rapid core-genome alignment and visualization of thousands of intraspecific microbial genomes. Genome Biol. 2014, 15, 524. [CrossRef]

32. Letunic, I.; Bork, P. Interactive tree of life (ITOL) v3: An online tool for the display and annotation of phylogenetic and other trees. Nucleic Acids Res. 2016, 44, W242-W245. [CrossRef] [PubMed]

33. Francisco, A.P.; Vaz, C.; Monteiro, P.T.; Melo-Cristino, J.; Ramirez, M.; Carriço, J.A. PHYLOViZ: Phylogenetic inference and data visualization for sequence based typing methods. BMC Bioinform. 2012, 13, 87. [CrossRef] [PubMed]

34. Page, A.J.; Cummins, C.A.; Hunt, M.; Wong, V.K.; Reuter, S.; Holden, M.T.G.; Fookes, M.; Falush, D.; Keane, J.A.; Parkhill, J. Roary: Rapid large-scale prokaryote Pan genome analysis. Bioinformatics 2015, 31, 3691-3693. [CrossRef] [PubMed]

35. Brynildsrud, O.; Bohlin, J.; Scheffer, L.; Eldholm, V. Rapid scoring of genes in microbial pan-genome-wide association studies with scoary. Genome Biol. 2016, 17, 238. [CrossRef] [PubMed] 